\title{
Role of two dimensionality in $\mathrm{MgB}_{2}$
}

\author{
W.E. Pickett ${ }^{\text {a,* }}$, J.M. An ${ }^{\text {a,b }}$, H. Rosner ${ }^{\text {a }}$, S.Y. Savrasov ${ }^{\text {c }}$ \\ a Department of Physics, University of California, Davis, CA 95616, USA \\ b Lawrence Berkeley National Laboratory, Berkeley, CA 94720, USA \\ c Department of Physics, New Jersey Institute of Technology, NJ 07102, USA
}

\begin{abstract}
In the theoretical study of the origin of superconductivity in $\mathrm{MgB}_{2}$, the importance of the two dimensionality of the electronic structure has not been clear. Here we use the related system, and predicted superconductor, $\mathrm{Li}_{1-x} \mathrm{BC}$ to illustrate the importance the two dimensionality of the $\sigma$ bands has for (1) the occurrence of high $T_{\mathrm{c}}$, (2) the possibility of raising the critical temperature, and (3) the lattice instabilities that accompany strong electron-phonon coupling.

(c) 2003 Elsevier Science B.V. All rights reserved.
\end{abstract}

Keywords: Superconductivity; $\mathrm{MgB}_{2}$; Two-dimensionality; Electron-phonon coupling

\section{Background: $\mathrm{MgB}_{2}$ and $\mathrm{Li}_{1-x} \mathrm{BC}$}

A good understanding of conventional 'high temperature superconductivity' [1] in intermetallic compounds $\left(T_{\mathrm{c}} \sim 20 \mathrm{~K}\right)$ resulted from intense research conducted in the 1960s and 1970s, and one feature that emerged was that cubic crystal structures were most favorable for high critical temperature. Examples are the A15 compounds ( $T_{\mathrm{c}}$ up to $23 \mathrm{~K}$ ) and $\mathrm{NbC}_{1-x} \mathrm{~N}_{x}$, with $T_{\mathrm{c}}$ near $17 \mathrm{~K}$. The discovery of $\mathrm{MgB}_{2}$ with $T_{\mathrm{c}}=40 \mathrm{~K}$ [2] has upset this and several other "understandings," including the need for high carrier density of states $N\left(E_{\mathrm{F}}\right)$ and open-shell transition metal atoms- $\mathrm{MgB}_{2}$ has neither. The theoretical work since January 2001 [3-8] has clarified many of the causes of this high $T_{\mathrm{c}}$ : strongly covalent-bonding states, nor-

\footnotetext{
${ }^{*}$ Corresponding author. Tel.: +1-530-752-0926; fax: +1-530752-4717.

E-mail address: pickett@physics.ucdavis.edu (W.E. Pickett).
}

mally fully occupied in covalently bonded materials, are driven to the Fermi level $\left(E_{\mathrm{F}}\right)$ by the chemistry of $\mathrm{MgB}_{2}$, and the resulting hole carriers are exceedingly strongly coupled to the bondstretching modes. This "covalent" coupling is what drives the critical temperature from zero (or near) to $40 \mathrm{~K}$.

The role of two dimensionality (2D), although noted many times, has remained less clear. This question is particularly important in view of relatively high temperature superconductivity discovered recently in other layered materials: $T_{\mathrm{c}}$ up to $25.5 \mathrm{~K}$ in electron-doped $\mathrm{HfNCl}$ [9], up to $14 \mathrm{~K}$ in $\mathrm{CaSi}_{2}$ [10] (which is isostructural with $\mathrm{MgB}_{2}$ ), and up to $11 \mathrm{~K}$ in $\mathrm{Y}_{2} \mathrm{C}_{2} \mathrm{I}_{2-x} \mathrm{Br}_{x}$ [11]. Here we clarify several aspects of electron-phonon coupling in a $2 \mathrm{D}$ electronic system, using the system $\mathrm{Li}_{1-x} \mathrm{BC}$ that lies within the same class of materials (Fig. 1) as $\mathrm{MgB}_{2}$ and has been predicted to have even stronger coupling than $\mathrm{MgB}_{2}[12,13]$. LiBC itself is a semiconductor, but hole doping should occur nearly rigid-band-like [13], leading to cylindrical 
(Li)
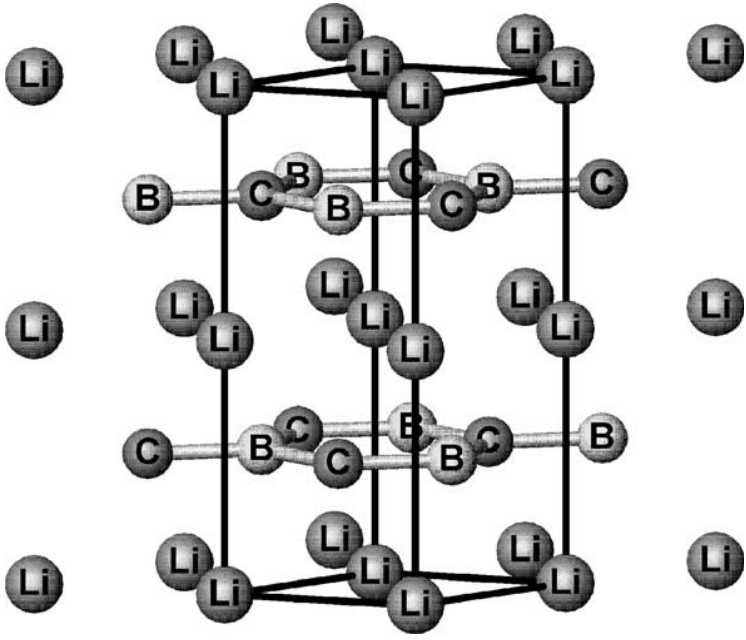

Fig. 1. Crystal structure of LiBC, illustrating the similarity to $\mathrm{MgB}_{2}$. The $\mathrm{B}-\mathrm{C}$ layers correpsond to the $\mathrm{B}-\mathrm{B}$ graphene layers of $\mathrm{MgB}_{2}$, and $\mathrm{Li}$ ions lie in the same interstitial positions. $\mathrm{B}$ and $\mathrm{C}$ alternate along the $\hat{c}$ axis, doubling the unit cell.

hole Fermi surfaces that are essentially like those that were predicted and then observed in $\mathrm{MgB}_{2}$ [15-17]. The strong electron-phonon coupling that accompanies the transition to the metallic state produces remarkable changes in the phonon spectrum, as calculated from density functional linear response theory and displayed in Fig. 2. A more thorough discussion of the phonon spectra and effects of electron-phonon coupling are presented elsewhere [13]. Here we concentrate on clarifying the role of two dimensionality.

\section{Importance of the -band two dimensionality}

The cylindrical $\sigma$ Fermi surfaces of $\mathrm{MgB}_{2}$ and hole-doped $\mathrm{LiBC}$ allow an analytic treatment of EP coupling that facilitates understanding. We consider a single Fermi surface. Phonon energies $\omega_{Q}(\hbar=1$, and including the branch index in the label $Q$ ) are given by

$\omega_{Q}^{2}=\Omega_{Q}^{2}+2 \Omega_{Q} \Pi\left(Q, \omega_{Q}\right)$,

where the unrenormalized frequency $\Omega_{Q}$ includes all self-energy effects except those arising from electron scattering within the $\sigma$ bands. The pho-
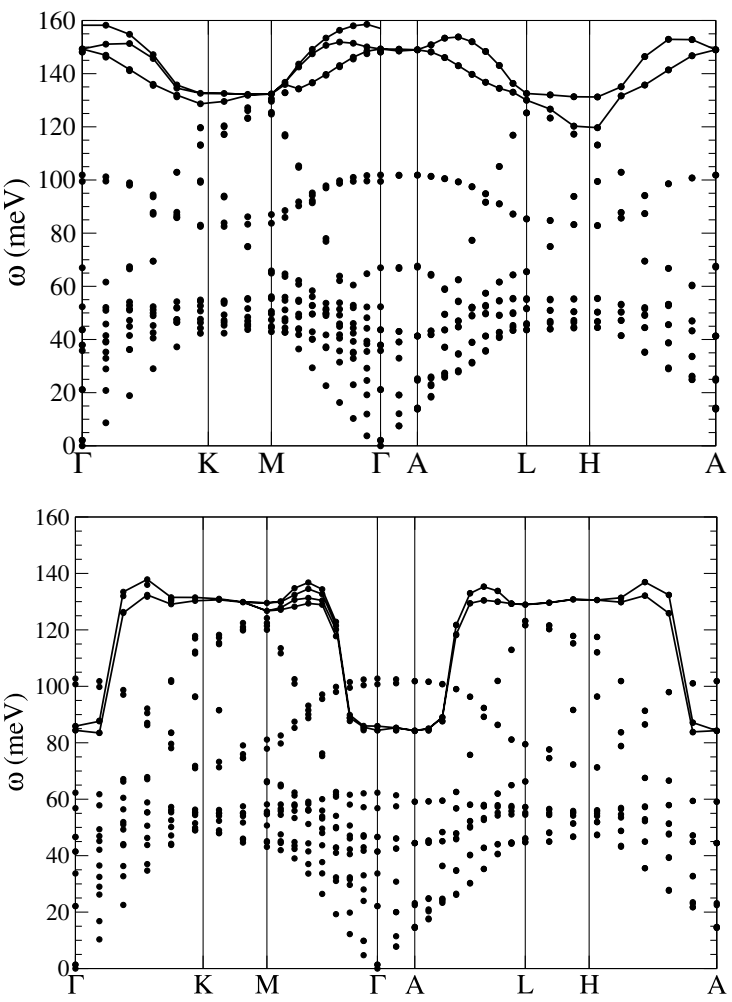

Fig. 2. Phonon dispersion curves from linear response calculations for (top) semiconducting $\mathrm{LiBC}$ and (bottom) strongly hole-doped and metallic $\mathrm{Li}_{0.75} \mathrm{BC}$, with bond-stretching modes connected by heavy lines. The important difference is the extremely strong renormalization downward $\left(\omega_{O}^{2}\right.$ decreases by $\sim 60 \%$ ) for $Q<2 k_{\mathrm{F}}$; the extreme van Hove singularities at $2 k_{\mathrm{F}}$ along $\Gamma-\mathrm{K}, \mathrm{M}-\Gamma, \mathrm{A}-\mathrm{L}, \mathrm{H}-\mathrm{A}$ are apparent.

non self-energy due to the $\sigma$ carriers (with dispersion $\left.\varepsilon_{k}=\left(k_{x}^{2}+k_{y}^{2}\right) / 2 m^{*}\right)$ is $\left(\eta_{Q} \equiv Q / 2 k_{\mathrm{F}}\right)$

$$
\begin{aligned}
\Pi(Q, \omega) & =-2 \sum_{k}\left|M_{k, Q}\right|^{2} \frac{f_{k}-f_{k+Q}}{\varepsilon_{k+Q}-\varepsilon_{k}-\omega-i \delta} \\
& \approx-2|M|^{2} \chi^{2 \mathrm{D}}(Q, \omega), \\
\chi^{2 \mathrm{D}}\left(Q, \omega_{Q}\right) & \approx N\left(\varepsilon_{\mathrm{F}}\right)+i \pi \omega_{Q}\left[\frac{4 \pi}{A_{\text {cell }}} \frac{N^{2}\left(\varepsilon_{\mathrm{F}}\right)}{\pi\left(2 k_{\mathrm{F}}^{2}\right)} \frac{1}{\eta \sqrt{1-\eta^{2}}}\right], \\
\eta & \equiv \frac{Q}{2 k_{\mathrm{F}}}<1, \\
& \approx N\left(\varepsilon_{\mathrm{F}}\right)\left[1-\sqrt{1-\eta^{-2}}\right], \quad \eta>1 .
\end{aligned}
$$

Here $f_{k} \equiv f\left(\varepsilon_{k}\right)$ is the Fermi occupation factor, a mean square matrix element has been extracted 
from the sum, and the usual adiabatic approximation has been made, leaving the self-energy in terms of the two dimensional Lindhard function $\chi_{L}^{2 \mathrm{D}}$ [18]. The density of states $N(\varepsilon)=A_{\text {cell }} m^{*} / 2 \pi$ per spin per unit 2D cell $\left(A_{\text {cell }}\right.$ is the basal plane area) is independent of energy in 2D. Since the unrenormalized optical modes should vary smoothly for $Q<2 k_{\mathrm{F}}$ and is flat for $Q \rightarrow 0$, and the two dimensional susceptibility is constant, the bond stretching (" $E_{2 g}$ ") frequencies with $Q<2 k_{\mathrm{F}}$ are renormalized to the nearly $Q$-independent value

$\omega_{2 g}^{2}=\Omega_{2 g}^{2}-4 N\left(\varepsilon_{\mathrm{F}}\right) \Omega_{2 g}|M|^{2}$.

Note that the softening is proportional to the product of the square of the deformation potential and the density of states (i.e. the effective mass), but it is independent of the doping level of $\sigma$ holes $n_{\sigma}\left(\propto k_{\mathrm{F}}^{2}\right)$. Hole doping changes the fraction of phonons that are renormalized $\left(E_{2 g}\right.$ derived modes with $Q<2 k_{\mathrm{F}}$ ) but does not affect the amount of softening.

The phonon line width $\gamma_{Q}$ is given by

$$
\begin{aligned}
\gamma_{Q} & =\frac{\Omega_{Q}}{\omega_{Q}}\left|\Im m \Pi\left(Q, \omega_{Q}\right)\right| \\
& =\pi \Omega_{Q}|M|^{2}\left[\frac{8 \pi}{A_{\text {cell }}} \frac{N^{2}\left(\varepsilon_{\mathrm{F}}\right)}{\pi\left(2 k_{\mathrm{F}}\right)^{2}} \frac{\Theta(\eta) \Theta(1-\eta)}{\eta \sqrt{\left(1-\eta^{2}\right)}}\right] .
\end{aligned}
$$

The divergence for $\eta \rightarrow 1\left(Q \rightarrow 2 k_{\mathrm{F}}\right)$ is integrable, and the singularity at $\eta \rightarrow 0$ (also integrable) is an artifact that can be handled directly when $Q \sim 0$ quantities are needed [19] but involves negligible volume. Note that the line width is proportional to the square of the effective mass, and to the square of the deformation potential, but in addition it is inversely proportional to the $\sigma$-hole doping level $\left(\propto k_{\mathrm{F}}^{2}\right)$. This singular behavior of the line width at low doping level may introduce new behavior as 2D materials are doped (viz. Li is extracted from LiBC) where non-adiabatic effects also become a factor.

Of central importance to the determination of $T_{\mathrm{c}}$ is the total $\lambda_{2 g}$ from the bond-stretching modes, and of course its variation with doping is of great interest. Since $\lambda$ is the average over all the modes of the crystal of the mode $\lambda\left(\lambda_{Q}\right)$, it can be expressed as the contribution from the strongly coupled and renormalized bond stretching and the rest: $\lambda=$ $\lambda_{2 g}+\lambda_{0}$. Using the expression for $\lambda_{Q}$ in terms of the line width [20], one finds for a hexagonal cell $\left(A_{\text {cell }}=(\sqrt{3} / 2) a^{2}\right)$

$$
\begin{aligned}
\lambda_{Q} & =\frac{2 N_{v}}{\pi N\left(\varepsilon_{\mathrm{F}}\right)} \frac{\gamma_{Q}}{\omega_{Q}^{2}} \\
& =\frac{8 \Omega_{Q}}{\sqrt{3} \omega_{Q}^{2}}|M|^{2} N_{v} \frac{N\left(\varepsilon_{\mathrm{F}}\right)}{\left(k_{\mathrm{F}} a\right)^{2}} \frac{\Theta(\eta) \Theta(1-\eta)}{\eta \sqrt{\left(1-\eta^{2}\right)}}, \\
\lambda_{2 g} & =\sum_{Q v} \lambda_{Q v} / \sum_{Q v} 1 \propto \frac{\left|M^{2}\right| N\left(\varepsilon_{\mathrm{F}}\right) \Omega_{2 g}}{\omega_{2 g}^{2}} .
\end{aligned}
$$

Here the average over $N_{v}$ phonon branches $v$ has been included explicitly in the notation.

Various results can be gathered:

- from Eq. (3), the amount of softening is independent of any explicit dependence on doping level,

- the line width $\gamma_{Q}$, and the mode $\lambda_{Q}$, increase without bound (as $k_{\mathrm{F}}^{-2}$ ) as the doping level (i.e. $\left.k_{\mathrm{F}}\right)$ is decreased,

- the fraction of phonons (proportional to $k_{\mathrm{F}}^{2}$ ) that are strongly coupled decreases with doping level,

- as a result, the total coupling strength $\lambda_{2 g}$ from the bond-stretching modes is independent of any explicit dependence on the doping level (via $k_{\mathrm{F}}$ ),

- the mean value of the mode $\lambda_{Q}$ for the softened $E_{2 g}$ modes, unlike the zone average, tends to diverge as the hole doping decreases,

- this divergence signals that Migdal theory for the coupled electron-phonon system is no longer justified for these renormalized phonons.

The usual criterion for failure of Migdal's theorem is losing the adiabatic approximation $\left(\omega_{2 g} / \varepsilon_{\mathrm{F}} \ll 1\right)$. Here the failure is different: due to the very large mode $\lambda$ 's the necessary condition $\lambda_{Q} \omega_{Q} / \varepsilon_{\mathrm{F}} \ll 1$ no longer holds, even when $\omega_{Q} / \varepsilon_{\mathrm{F}}$ is not strongly violated.

Applying these relations and using values estimated from Fig. 2: $\Omega_{2 g} \approx 140 \mathrm{meV}, \omega_{2 g} \approx 90 \mathrm{meV}$, one obtains an average mode coupling strength for $E_{2 g}$ phonons with $\eta<1$ is at least 25 . Since only 4/ 18 branches are strongly coupled, and only about 
$7.5 \%$ of the zone lies in the region $\eta<1$, the contribution to $\lambda$ from the strongly coupled $E_{2 g}$ modes is roughly $25 \times 4 / 18 \times 0.075 \approx 0.4$, very consistent with the value obtained from linear response calculations of $\alpha^{2} F$ from these modes. The mean mode $\lambda$ from all other phonons (equal to their contribution to $\lambda$ since it includes $98 \%$ of all modes) also is $\approx 0.4$.

The phonon relative line width is given by [20] $\gamma_{Q} / \omega_{Q}=\lambda_{Q} \pi N(0) \omega_{Q} / 4 \sim 1 / 2$, where the calculated $N\left(\varepsilon_{\mathrm{F}}\right)=0.28 \mathrm{eV}^{-1}$ per unit cell per spin for the $\sigma$ bands has been used. This result $\gamma_{Q} \sim \omega_{Q}$ for the $E_{2 g}$ branch with $Q \leqslant 2 k_{\mathrm{F}}$ is crude but not far from the experimental values [21,22] (Raman line width of $\mathrm{MgB}_{2}$ equal to $1 / 3$ to $1 / 2$ of the peak energy) and reflects the fact that this phonon branch is so ill-defined from extremely strong EP coupling that conventional phonon theory, and thus all of the expressions above, are becoming suspect.

Now we summarize and discuss some implications of these results. Use of $\mathrm{Li}_{1-x} \mathrm{BC}$ has allowed us to identify and quantify the extreme phonon softening arising from the ultrastrong EP coupling to $E_{2 g}$ modes; this behavior occurs also in $\mathrm{MgB}_{2}$. Mode $\lambda_{2 g}^{\prime} \mathrm{s} \geqslant 25$ and line widths comparable to the frequency point to inadequacies of standard (Migdal) EP theory for these systems. The widely discussed polaronic effects in quasi-2D systems $[23,24]$ are not evident in ARPES [14] and dHvA [15] data, which reflect bandlike quasiparticles both at high energy and low energy. In the optic phonon range, however, the dynamical behavior of the system must be very complex, and may be at the root of the confusing optical conductivity data [25]. Peaking of the interaction strength at $2 k_{\mathrm{F}}$ suggests polaronic correlations that are most pronounced at wavelength $2 \pi / 2 k_{\mathrm{F}} \approx 6 a, \omega \sim 55$ $\mathrm{meV}$, in $\mathrm{Li}_{0.75} \mathrm{BC}$; bond-stretching modes with wavelengths longer than this are ill defined and will couple to other modes.

This extremely strong, and abruptly $Q$ dependent, EP coupling provides new insight into the controversy about limits on $T_{\mathrm{c}}$ from lattice instabilities [26]. Any tendency toward higher $T_{\mathrm{c}}$ for higher doping level in $\mathrm{Li}_{1-x} \mathrm{BC}$ will not be due simply to higher carrier concentration, as there is no $k_{\mathrm{F}}$ dependence of $\lambda_{2 g}$ in Eq. (5). Although " $N(\varepsilon)$ is constant" is a good guideline, actually $N\left(\varepsilon_{\mathrm{F}}\right)$ can

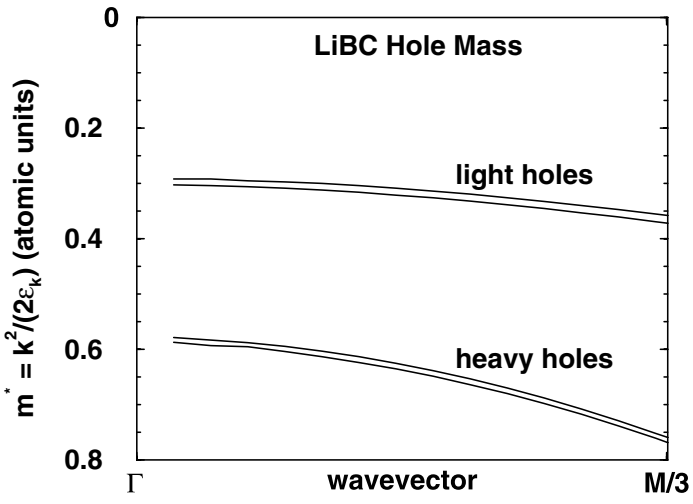

Fig. 3. Calculated effective band mass $m^{*}\left(k_{\mathrm{F}}\right)$ defined by $\varepsilon_{k}=k^{2} /\left.2 m^{*}(k)\right|_{k_{\mathrm{F}}}$, for $\mathrm{Li}_{1-x} \mathrm{BC}$. The notation " $\mathrm{M} / 3$ " denotes a distance in the Brillouin zone one third of the way to the $\mathrm{M}$ point. The small splitting of the dispersion of both light and heavy holes indicates how small the interlayer interaction (one measure of non-2D character) is in $\mathrm{Li}_{1-x} \mathrm{BC}$.

change due to non-parabolicity of the dispersion relation. Fig. 3 shows the $k_{\mathrm{F}}$ dependence of the effective mass $m^{*}\left(k_{\mathrm{F}}\right)$ (see caption) for $\mathrm{Li}_{1-x} \mathrm{BC}$, and it can be seen that the resulting increase in $\lambda_{2 g}$ with doping can be important. Increased doping, and the larger value of $k_{\mathrm{F}}$, means that more phonons are renormalized, but the total coupling strength $\lambda_{2 g}$ is redistributed over these additional softened phonons. For low doping level, the increasingly large coupling may impact crystal stability directly; of course, non-adiabatic corrections must be included in the theory in this regime. A way to try to increase $T_{\mathrm{c}}$ would be by increasing the deformation potential (by choice of constituent atoms or layered structure) or by increasing $m^{*}$ (possibly by pressure); such changes would also increase the softening and move the system closer to instability as conventional theory would predict. We remind that, in 2D, all of the phonons are renormalized by the same amount - no single $Q$ vector is singled out for instability. These two mechanisms of increasing $\lambda$ illustrate the importance of the $Q$-distribution of coupling strength for (in)stability.

\section{Acknowledgements}

W.E.P. acknowledges useful discussions with I.I. Mazin and O.K. Andersen related to mode $\lambda$ 's 
in $\mathrm{MgB}_{2}$. This work was supported by National Science Foundation Grant DMR-0114818, and by the Deutscher Akademischer Austauschdienst.

\section{References}

[1] P.B. Allen, B. Mitrović, in: Solid State Physics, vol. 37, Academic, New York, 1982, pp. 2-92.

[2] J. Nagamatsu et al., Nature 410 (2001) 63.

[3] J.M. An, W.E. Pickett, Phys. Rev. Lett. 86 (2001) 4366.

[4] J. Kortus et al., Phys. Rev. Lett. 86 (2001) 4656.

[5] Y. Kong et al., Phys. Rev. B 64 (2001) 020501.

[6] K.-P. Bohnen, R. Heid, B. Renker, Phys. Rev. Lett. 86 (2001) 5771.

[7] T. Yildirim et al., Phys. Rev. Lett. 87 (2001) 037001.

[8] A. Liu, I.I. Mazin, J. Kortus, Phys. Rev. Lett. 87 (2001) 087005.

[9] S. Yamanaka, Ann. Rev. Mater. Sci. 30 (2000) 53, and references therein.

[10] S. Sanfilippo et al., Phys. Rev. B 61 (2000) R3800.

[11] A. Simon et al., Z. Anorg. Allg. Chem. 622 (1996) 123.

[12] H. Rosner, A. Kitaigorodsky, W.E. Pickett, Phys. Rev. Lett. 88 (2002) 127001.

[13] J.M. An, H. Rosner, S.Y. Savrasov, W.E. Pickett, condmat/0207542.
[14] H. Uchiyama et al., Phys. Rev. Lett. 88 (2002) 157002.

[15] E.A. Yelland et al., Phys. Rev. Lett. 88 (2002) 217002.

[16] I.I. Mazin, J. Kortus, Phys. Rev. B 65 (2002) 180510.

[17] H. Rosner, J.M. An, W.E. Pickett, S.-L. Drechsler, Phys. Rev. B 66 (2002) 024521.

[18] J.G. Kim et al., Phys. Rev. B 59 (1999) 3661.

[19] C.O. Rodriguez et al., Phys. Rev. B 42 (1990) 2692.

[20] P.B. Allen, Phys. Rev. B 6 (1972) 2577; P.B. Allen, M.L. Cohen, Phys. Rev. Lett. 29 (1972) 1593 ;

A numerical correction is given in Eq. (4.27) of P.B. Allen, in: G.K. Horton, A.A. Maradudin (Eds.), Dynamical Properties of Solids, North-Holland, Amsterdam, 1980 (Chapter 2).

[21] H. Martinho et al., cond-mat/0105204.

[22] J.W. Quilty et al., Phys. Rev. Lett. 88 (2002) 087001.

[23] R. Micnas, J. Ranninger, S. Robaszkiewicz, Rev. Mod. Phys. 62 (1990) 113.

[24] K. Nasu, Phys. Rev. B 35 (1987) 1748.

[25] J.J. Tu et al., Phys. Rev. Lett. 87 (2001) 277001.

[26] M.L. Cohen, P.W. Anderson, in: D.H. Douglass (Ed.), Superconductivity in d- and f-Band Metals, AIP, New York, 1972;

D.A. Kirzhnits et al., J. Low Temp. Phys. 10 (1973) 79;

P.B. Allen, M.L. Cohen, D.R. Penn, Phys. Rev. B 38 (1988) 2513. 